\title{
Metástases dos ossos gnáticos: estudo retrospectivo de 10 casos
} retrospective study of 10 cases

\author{
Antonio Azoubel Antunes', Antonio Pessoa \\ Antunes $^{2}$
}

Palavras-chave: mandíbula, maxila, metástase neoplásica, neoplasias de cabeça e pescoço, neoplasias ósseas. Keywords: mandible, maxilla, neoplasm metastasis, head and neck neoplasms, bone neoplasms.

\section{Resumo / Summary}

\begin{abstract}
$\mathrm{M}$ etástases em cavidade oral são extremamente raras, representando $1 \%$ de todas as neoplasias malignas orais. Objetivo: Analisar retrospectivamente a incidência de metástase nos maxilares, traçando seu perfil de comportamento epidemiológico. Material e Métodos: Através de estudo retrospectivo, foram analisados o sexo, idade, localização topográfica da metástase, sítio do tumor primário e tipo histológico, no período de janeiro de 1980 a janeiro de 2000, de um total de 10 pacientes. Resultados: Observou-se que 5 pacientes eram do sexo masculino (50\%), 3 casos ocorreram nas $2^{\mathrm{a}}$ e $4^{\mathrm{a}}$ décadas de vida $(30$ e $30 \%)$. Concernente à localização topográfica da metástase, metade dos casos ocorreu na maxila e metade na mandíbula. A tireóide e próstata foram os sítios de tumor primário que mais metastizaram para os ossos gnáticos (3 casos cada) e o adenocarcinoma foi o tipo histológico de maior ocorrência de metástase (50\%). Conclusões: As metástases nos maxilares acometem igualmente faixa etária, sexo e localização topográfica. A tireóide e próstata parecem ser os sítios mais freqüentes de metástase à distância, e o adenocarcinoma o tipo histológico de maior incidência de metástase.
\end{abstract}

\begin{abstract}
$\mathrm{O}$ ral cavity metastases are extremely rare, and comprise $1 \%$ of all mouth malignant neoplasms. Aim: to retrospectively analyze the incidence of maxillary metastases, and to trace its epidemiological behavior. Material and Methods: A retrospective study was done of the period between January 1980 and January 2000; the following indicators were reviewed: sex, age, site of the metastasis, site of the primary tumor, and the histological type of tumors in 10 patients. Results: The prevalence was similar in males and females ( 05 cases - 50\%); three cases presented in the 2nd and 4 th decades of life $(30-30 \%)$. About half of the cases of metastases occurred in the maxilla and half in the mandible. The thyroid and prostate glands were the most prevalent primary tumor sites for metastases (03 cases each); the adenocarcinoma histological type had the highest metastatic rate (50\%). Conclusions: Metastasis of the jaws is very rare and may occur at any age in both the genders, where the prevalence is equal for each sex. The thyroid and prostate seem to be the most frequent sites of distance metastasis of the jaws; the adenocarcinoma is the most frequent histological type causing such metastases.
\end{abstract}

\footnotetext{
${ }^{1}$ Cirurgião-dentista, Aluno do curso de especialização em Cirurgia e Traumatologia Buco-Maxilo-Facial ${ }^{2}$ Cirurgião de Cabeça e Pescoço.

Centro de Oncologia (CEON) do Hospital Universitário Oswaldo Cruz (HUOC) da Universidade de Pernambuco (UPE). Endereço para correspondência: Antonio Azoubel Antunes - Rua Guilherme Pinto 345/303 Derby Recife PE 52010-210. E-mail: antunesctbmf@yahoo.com.br

Este artigo foi submetido no SGP (Sistema de Gestão de Publicações) da RBORL em 30 de março de 2007. cod. 3911. Artigo aceito em 13 de maio de 2007.
} 


\section{INTRODUÇÃO}

As metástases em cavidade oral são extremamente raras, e representam 1\% de todas as neoplasias malignas da boca. Localizam-se em 80 a 90\% dos casos na mandíbula, sendo ainda mais raras em maxila. Podem surgir de várias neoplasias malignas primárias, localizadas principalmente em mama, próstata, pulmão, tireóide e rim, que são as que dão origem à maioria das metástases ósseas ${ }^{1}$.

O comprometimento metastático dos referidos ossos apresenta uma variedade de sinais e sintomas, podendo, entretanto, o paciente encontrar-se totalmente assintomático, e o diagnóstico ter sido feito mediante um achado de um exame radiológico.

Os tumores da mandíbula e da maxila, pela sua evolução natural e níveis de comprometimento loco-regional, determinam alterações morfológicas e funcionais significativas, exigindo, na maioria das vezes, uma abordagem multidisciplinar completa ${ }^{2}$.

O diagnóstico das referidas neoplasias pode, entretanto, oferecer dificuldades com relação aos seus aspectos diferenciais. A precisão de exames laboratoriais, como a imunohistoquímica, pode oferecer alguns destes parâmetros. O tipo de parestesia, seja unilateral, hipoestesia ou anestesia do lábio inferior, com ou sem presença de dor, pode ser a manifestação clínica inicial de um percentual significativo de lesões que comprometem o osso mandibular. Alterações de sensibilidade envolvendo o lábio inferior, até sem importantes mudanças radiológicas, devem alertar o especialista em pensar na possibilidade de uma metástase inicial ${ }^{3}$.

As metástases ósseas são achados relativamente comuns nos carcinomas avançados, especialmente aqueles que se originam da mama, pulmão, tireóide, próstata e rim.
Quando os ossos gnáticos são envolvidos, a mandíbula tem uma predileção em relação à maxila na proporção de 4:1, particularmente em sua porção posterior, onde existe abundante tecido vascular e hematopoiético ${ }^{3}$.

O presente estudo tem como objetivo analisar retrospectivamente a incidência de metástase nos maxilares, em um período de 20 anos, de pacientes atendidos e tratados em serviço de referência, traçando o perfil de comportamento epidemiológico a partir dos dados obtidos na amostra.

\section{MATERIAL E MÉTODOS}

Foi realizado um estudo retrospectivo no período de janeiro de 1980 a dezembro de 2000, dos pacientes atendidos e tratados no ambulatório de Cirurgia de Cabeça e Pescoço do Centro de Oncologia (CEON) do Hospital Universitário Oswaldo Cruz (HUOC) - Universidade de Pernambuco (UPE).

No período supracitado, encontrou-se registrados 190 casos de tumores ósseos do segmento cabeça e pescoço, dos quais dez pacientes $(5,2 \%)$ eram portadores de neoplasias metastáticas dos maxilares, sendo seis mulheres e quatro homens, com idade entre 13 e 75 anos (média de 43 anos). Foram analisados ainda a localização topográfica da metástase, sítio do tumor primário e tipo histológico do tumor (Tabela 1).

O presente estudo foi cadastrado e devidamente aprovado no Comitê de Ética da referida instituição sob o protocolo nำ134707/07.

\section{RESULTADOS}

De acordo com os dados obtidos, os pacientes da amostra foram assim distribuídos:

Tabela 1. Características dos pacientes.

\begin{tabular}{cccccc}
\hline Paciente & Gênero & Idade & Localização & Sítio tumor primário & Tipo histológico \\
\hline 1 & F & 13 & Maxila & Tireóide & Adenocarcinoma papilífero \\
2 & F & 64 & Mandíbula & Tireóide & Carcinoma folicular \\
3 & M & 75 & Mandíbula & Próstata & Adenocarcinoma S.O.E. \\
4 & F & 62 & Mandíbula & Mama & Adenocarcinoma mal-diferenciado \\
5 & F & 75 & Mandíbula & Partes Móide & Carcinoma folicular \\
6 & M & 19 & Maxila & Próstata & Sarcoma alveolar \\
7 & M & 38 & Próstata & Adenocarcinoma S.O.E. \\
8 & M & 32 & Maxila & Mama & Adenocarcinoma S.O.E. \\
9 & F & 36 & Maxila & Partes Moles & Farcinoma ductal \\
10 & F & 16 & Mandíbula & & \\
\hline
\end{tabular}

Abreviações: S.O.E., Sem outra especificação. 


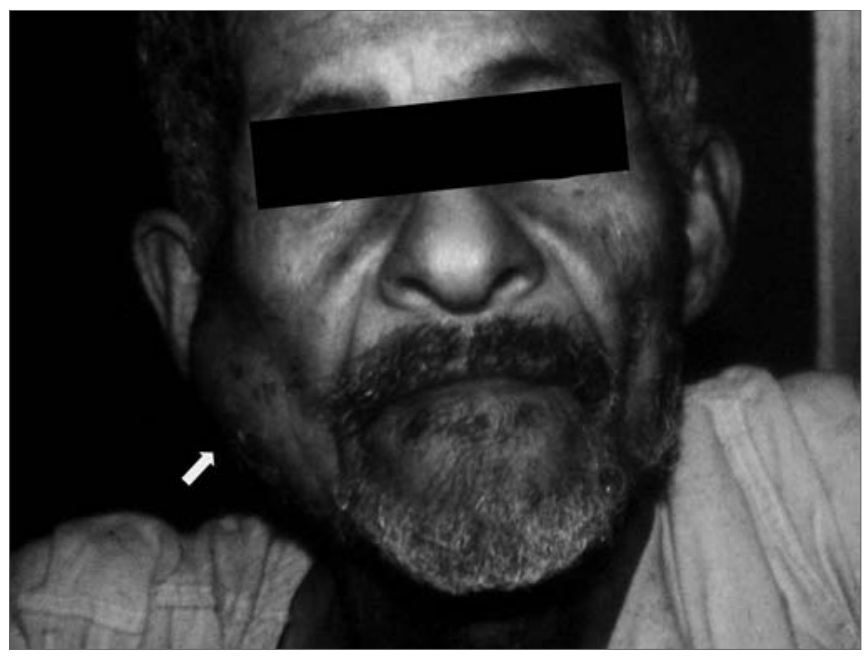

Figura 1. Aspecto clínico de metástase de adenocarcinoma de próstata em mandíbula.

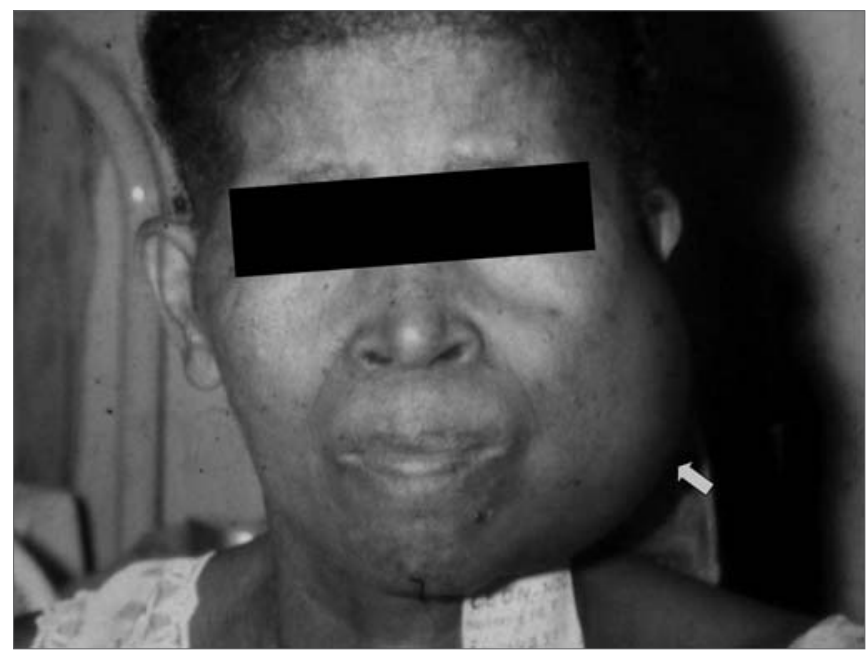

Figura 2. Aspecto clínico de metástase de adenocarcinoma de mama em mandíbula.

\section{DISCUSSÃO}

As neoplasias malignas dos ossos gnáticos, particularmente da mandíbula, têm uma incidência percentual mínima em relação à totalidade dos tumores humanos (menos de $0,7 \%$ ). As referidas neoplasias, pela sua evolução, níveis de comprometimento loco-regional e as formas agressivas de tratamento, acarretam alterações morfológicas, funcionais e estéticas significativas. São divididos em malignos primários e secundários ou metastáticos. Sob o ponto de vista histológico, apresentam três origens: os derivados do tecido odontogênico, dos tecidos conjuntivos esqueletogênicos e os de outros tecidos não-odontogênicos e não-esqueletogênicos ${ }^{2}$.

Os ossos gnáticos normalmente são considerados localizações incomuns para metástase, podendo, entretanto, estar envolvidos com uma maior freqüência do que relatado geralmente na literatura ${ }^{4}$.
Rutsatz et al. ${ }^{5}$, em um período de 35 anos, relatam 1008 pacientes portadores de tumores malignos da face (partes moles e ossos) e da boca, encontrou apenas cinco pacientes $(0,5 \%)$ com neoplasias metastáticas para a mandíbula, referidas como metástases à distância de tumores de diversas localizações topográficas.

Pruckmayer et al. ${ }^{6}$ realizaram um importante estudo retrospectivo em 763 pacientes apresentando dores inespecíficas na mandíbula, dos quais, apenas nove pacientes $(1,2 \%)$ apresentaram metástases para a mandíbula. O autor alerta que o fator dor é um indicador importante para a investigação, e que quando as metástases ocorrem nos ossos gnáticos traduzem doença disseminada ${ }^{4,6}$, fato este também observado na amostra do presente trabalho.

Analisando as faixas etárias dos pacientes, verificouse que 3 casos ocorreram nas $2^{\underline{a}}$ e $4^{\underline{a}}$ décadas de vida (30\%), enquanto 2 casos ocorreram nas $7^{\text {a }}$ e $8^{\underline{a}}$ décadas (20\%). Os extremos etários variaram entre 13 e 75 anos (mediana de 43 anos).

Van der Waal et al. ${ }^{7}$, analisando uma amostra de 1537 casos de câncer de boca, dos quais 24 sendo tumores metastáticos, observaram igual distribuição por sexo e idade, com extremos etários de 8 a 90 anos, e uma mediana de 60 anos de idade.

D'Silva et al. ${ }^{8}$ comentam em sua amostra a distribuição por sexo e faixa etária das metástases dos maxilares, afirmando que as mulheres são acometidas pelo dobro do número de metástases que os homens, com faixa etária de 31 a 40 anos e 71 a 80 anos, respectivamente, como de maior ocorrência, confrontando com os dados obtidos na presente pesquisa.

Concernente à localização topográfica, a mandíbula e a maxila apresentaram igual acometimento de metástases ósseas, com 5 casos cada, contrariando o que relata a literatura, em que aponta em média uma prevalência de $80 \%$ das metástases ósseas para o osso mandibular ${ }^{4,9}$.

D'Silva et al. ${ }^{8}$, em estudo retrospectivo de 114 casos de doença metastática nos maxilares, relataram que a predileção pelo osso mandibular é mais freqüente no sexo feminino, quando comparado ao masculino.

Hirshberg et al. ${ }^{10}$ realizaram um estudo de 390 casos de tumores metastáticos para os ossos gnáticos, onde 316 casos (81\%) estavam localizados na mandíbula, e apenas 58 casos (14\%) ocorreram na maxila; ambos os ossos foram envolvidos em 21 pacientes (5,4\%).

Com relação aos tipos histológicos dos tumores metastáticos, o material em estudo mostrou o adenocarcinoma papilífero (um caso), adenocarcinoma mal diferenciado (um caso), adenocarcinoma S.O.E. (três casos), carcinoma folicular (dois casos), fibrossarcoma mixóide (um caso), o sarcoma alveolar (um caso), além do carcinoma ductal (um caso)

Van der Waal et al. ${ }^{7}$, apontam o adenocarcinoma como o principal tipo histológico responsável por metástases para ossos gnáticos, a partir das mais diversas 
localizações de tumores primários, corroborando com os dados obtidos na amostra.

De acordo com os dados obtidos, a localização primária da neoplasia que originou a metástase à distância para os ossos gnáticos foi representada pela glândula tireóide ( 3 casos), próstata ( 3 casos), como os sítios mais prevalentes, seguido da mama (2 casos) e partes moles (sarcomas) (2 casos).

As metástases para os tecidos moles da cavidade oral são incomuns, mas de ocorrência significativa. Dentre as neoplasias primárias mais comumente responsáveis por metástases à distância por via hematogênica para os ossos gnáticos, a literatura cita a mama, tireóide e pulmão $\mathrm{O}^{3,11,12}$. Outras localizações topográficas como próstata, partes moles e rim são consideradas $\operatorname{raras}^{3}$, e algumas que serão citadas mais adiante, raríssimas.

Piattelli et al. ${ }^{13}$, em 390 casos de câncer oral, encontraram 22 casos $(5,6 \%)$ de tumores metastáticos em ossos gnáticos, sendo o sítio primário a glândula prostática. Ressaltam, ainda, a rara ocorrência da mesma como sítio primário de tumores metastáticos dos maxilares.

Stavropoulos e Ord $^{14}$ referem um caso de uma paciente que apresentou metástase de câncer de mama para o côndilo mandibular com subseqüente fratura patológica, cujo caso foi inicialmente diagnosticado e tratado como uma manifestação não-neoplásica da articulação têmporo-mandibular.

Porter et al. ${ }^{15}$ referem um caso de teratoma maligno do testículo com metástase para ambos os côndilos mandibulares, cujo diagnóstico só foi feito em decorrência do paciente apresentar queixas de dor intensa nos locais afetados. Schumacher e Rohde ${ }^{16}$ reportam um raro caso de carcinoma da vulva com metástase para a mandíbula. Yanagi et al. ${ }^{11}$ fazem referência a um caso de um paciente do sexo masculino, com 59 anos de idade, portador de feocromocitoma maligno, com metástase para a mandíbula de um tumor primário de glândula adrenal direita. Ogunsalu e Samith ${ }^{17}$ relatam um caso de um neuroblastoma da adrenal direita com metástase para a mandíbula em um paciente do sexo masculino com 21 anos de idade. Localizações topográficas como partes moles (sarcomas) e tumores primitivos de outros ossos, raramente metastizam para a mandíbula e maxila ${ }^{4}$.

Existem ainda os casos de neoplasias de partes moles do pescoço, as quais apresentam um crescimento e comprometimento por contigüidade de estruturas vizinhas e o exame radiológico simular um tumor primitivo do osso. Captier et al. ${ }^{18}$ referem um caso de uma criança com 10 anos de idade, que apresentava um tumor no ramo horizontal da mandíbula, estendendo-se à região submandibular. O exame radiológico sugeria uma neoplasia primária do osso, porém o resultado do exame histopatológico mostrou tratar-se de um sarcoma sinovial de partes moles de pescoço, invadindo a mandíbula e o soalho da boca, embora seja um tumor de origem mesenquimal extremamente raro no segmento cefálico em crianças.

A literatura afirma que, em aproximadamente 30\% dos casos, pacientes portadores de metástase em ossos gnáticos possuem um tumor primário não diagnosticado

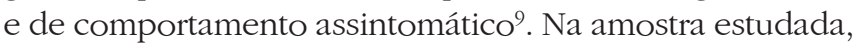
não foi observado nenhum caso de tumor primário oculto com metástase já existente.

O prognóstico para as neoplasias metastáticas dos ossos gnáticos é considerado reservado e sombrio, em decorrência da fisiopatologia do tumor primário que originou a metástase, que geralmente apresenta-se com alto grau de agressividade histológica, ocorrem em um estágio avançado da doença e pela existência das síndromes paraneoplásicas associadas.

\section{CONCLUSÕES}

1. As metástases em ossos gnáticos são raras e podem acometer qualquer faixa etária e ambos os sexos, com igual prevalência tanto para a mandíbula quanto para a maxila.

2. As glândulas tireóide e próstata parecem ser os sítios mais freqüentes de metástase à distância para ossos gnáticos, bem como o adenocarcinoma o tipo histológico de maior incidência de metástase.

\section{REFERÊNCIAS BIBLIOGRÁFICAS}

1. Jiménez JS, Blanco FA, Arévalo RE, Martinez MM. Metástasis en hueso maxilar superior de adenocarcinoma de esófago. Presentación de un caso clínico. Med Oral Patol Oral Cir Bucal 2005;10:252-7.

2. Perdicaris AAM. Tumores malignos da mandíbula. In: Brandão LG, Ferraz AR. Cirurgia de Cabeça e Pescoço - Princípios Básicos. 1aㅡ ed. São Paulo: Roca; 1989. p.321-8.

3. Amonet MR, Jackson MS, McGinnis JP, et al. Metastasis to the mandible - Case presentation. MDAJ 1998;54(2).

4. Neville BW, Damn DD, Allen CM e Bouquot JE. Patologia Oral e Maxilofacial. Guanabara Koogan; 1998. p. 431-80.

5. Rutsatz K, Peter U, Beust M, Hingst V. Metastasis of bronchial carcinoma in the temporomandibular joint. Case report. Dtsch Stomatol 1990;40(11):477-9.

6. Pruckmayer M, Glaser C, Marosi C, Leitha T. Mandibular pain as the leading clinical symptom for metastatic disease - Nine cases and review of the literature. Ann Oncol 1998;9(5):559-64.

7. Van der Waal RI, Buter J, Van der Waal I. Oral metastases: report of 24 cases. Br J Oral Maxillofac Surg 2003;41(1):3-6.

8. D'Silva NJ, Summerlin DJ, Cordell KG, et al. Metastatic tumors in the jaws: A retrospective study of 114 cases. J Am Dent Assoc. 2006;137(12):1667-72.

9. Bodner L, Geffen DB. Metastatic tumor of the jaw-diagnosis and management. Refuat Hapeh Vehashinayim 2003;20(1):59-61,81.

10. Hirshberg A, Leibovich P, Buchner A. Metastatic tumors to the jaw bones - Analysis of 390 cases. J Oral Path Med 1994;23:337-41.

11. Yanagi Y, Asaumi J, Hisatomi M, et al. Mandibular metastasis presenting as the initial manifestation of malignant pheochromocytoma. Eur J Radiol 2002;44(1):5-9.

12. Ueta E, Yoneda K, Ohno A, Osaki T. Intraosseous carcinoma arising from mandibular ameloblastoma with progressive invasion and pulmonary metastasis. Int J Oral Maxillofac Surg 1996;25(5):370-2.

13. Piattelli A, Fioroni M, Rubini C. Gingival metastasis from a prostate ade- 
nocarcinoma: report of a case. J Periodontol 1999 Apr;70(4):441-4. 14. Stavropoulos MF, Ord RA. Lobular adenocarcinoma of breast metastatic to the mandibular condyle. Report of a case and review of the literature. Oral Surg Oral Med Oral Pathol 1993;75(5):575-8.

15. Porter SR, Chandhry Z, Griffiths MJ, Scully C, Kabala J, Whipp E. Bilateral metastatic spread of testicular teratoma to mandibular condyles. Eur J Cancer B Oral Oncol 1996;32(5):359-61.

16. Schumacher A, Rohde E. Rare metastasis of vulvar cancers. Zentralb]
Gynakol 1992;114(7):379-81.

17. Ogunsalu C, Samith NJ. Metastatic neuroblastoma of the mandible mimicking osteogenis sarcoma radiologically. Case report. Aust Dent J 1999;44(3):207-10

18. Captier G, Montoya P, Duche R, et al. Synovial sarcoma of the mandible in children. Apropos of a case. Rev Stomatol Chir Maxillofac 1999;100(4):187-91. 